\title{
Ageing mechanism and mechanical degradation behaviour of polychloroprene rubber in a marine environment: Comparison of accelerated ageing and long term exposure
}

\author{
P.Y. Le Gac ${ }^{a, *}$, V. Le Saux ${ }^{b}$, M. Paris ${ }^{c}$, Y. Marco ${ }^{b}$
}

\author{
a IFREMER (French Ocean Research Institute), Centre de Brest, Materials and Structures Group, BP 70, F-29280 \\ Plouzané, France \\ ${ }^{\mathrm{b}}$ ENSTA Bretagne Laboratoire Brestois de Mécanique et des Systèmes EA 4325, 2 rue François Verny, F-29806 \\ Brest Cedex 9, France \\ ${ }^{c}$ Institut des Matériaux Jean Rouxel (IMN), Université de Nantes, CNRS, 2 rue de la Houssinière, BP 32229, \\ 44322 Nantes Cedex 3, France
}

*: Corresponding author : Pierre-Yves Le Gac,Tel.: +33 298224885 ; email address : pierre.yves.le.gac@ifremer.fr

\begin{abstract}
:
Polymers are widely used in marine environments due to their excellent properties and good weathering resistance. Despite this extensive use, their long term behaviour in such an aggressive environment is still not well known. To assess the polymer durability within reasonable durations, it is essential to perform accelerated ageing tests to accelerate the degradation kinetics but without any modification of the degradation process. This study therefore proposes and validates accelerated ageing tests to study marine ageing of a silica-filled chloroprene rubber (CR) used for offshore applications. Several accelerated ageing protocols are investigated for temperatures ranging from 20 to $80^{\circ} \mathrm{C}$ in renewed natural seawater. The ageing consequences are characterized using physical measurements (FTIR, solid state NMR) and mechanical testing based on monotonic tension tests. Instrumented micro-indentation tests are also employed, in order to describe accurately the ageing gradients through sample thickness. The measurements obtained on the samples cut from accelerated specimens are compared to those obtained from the topcoat of an offshore flowline aged under service conditions for 23 years. For both kinds of specimens, polychloroprene develops rapid material changes most clearly represented by a considerable increase in stiffness, which allows the accelerated ageing protocols to be validated.
\end{abstract}

Keywords: Chloroprene rubber ; Degradation ; Sea water ; Silica ; Accelerated ageing ; Natural ageing 


\section{Introduction}

The assessment of the durability of elastomeric products subjected to marine environment is a major issue for several industrial areas (offshore oil and gas industries, harbour and naval applications, renewable marine energy...) and is also necessary for new product certification and for better evaluation of maintenance periods and total lifetime. Polymer lifetime prediction in an aggressive medium can be considered as a three step process. First, the ageing mechanisms observed for natural ageing must be identified. Then, accelerated ageing tests are performed (in order to reduce test time) and the kinetics of the ageing reactions are investigated. These tests have to accelerate the degradation without modifying the mechanisms involved. Finally, a time-temperature superposition rule is applied in order to evaluate the ageing consequences under service conditions. The two main points to be checked are therefore the design of appropriate accelerated ageing tests, on the basis of well identified mechanisms, and an evaluation of the ageing gradients through the thickness in order to check if the measurements achieved on thin samples in the laboratories can be extended to large industrial structures. It is striking to observe that although these points have been extensively investigated for thermal ageing of organic materials, this is not the case for marine ageing. Even if marine ageing of organic materials has been widely investigated [1], [2], [3] and despite many marine applications (wetsuits, harbour drydock seals,...) very few papers investigate the ageing mechanisms of marine environment on elastomers [4], [5], [6], [7], [8]. This is probably partly due to the complexity of the degradation mechanisms in such materials but also because the property losses during ageing are often quite acceptable when the material is well formulated. The influence of the marine environment is complex, and depends clearly on the compound used. A first effect is the absorption of water by rubber, which induces a reversible change [9]. Other ageing mechanisms can involve hydrolysis [10], [11], additive extraction [12] and oxidation [13] but there are very few long term data available, which limits clear explanations of the ageing mechanisms involved. Dealing with polychloroprene (CR), the accelerated protocols that are proposed classically consist of raising the temperature, for environments 
ranging from air [14], [15] to synthetic [16], [17] or natural sea water [18]. Although chloroprene does not undergo hydrolysis [19], a considerable decrease of the tensile product (ultimate tensile strain multiplied by ultimate tensile stress) is observed during accelerated ageing. This consequence is observed for ageing in salt water [18] as well as in synthetic sea water for temperatures of around $40^{\circ} \mathrm{C}[20]$ or $70^{\circ} \mathrm{C}$ [17] and on specimens considered fully saturated in water, which indicates that the reversible water effect is not the only degradation involved. The formulation of the polychloroprene rubber also modifies the ageing behaviour of the material and the property loss is more significant for silica-filled compounds [20]. Nevertheless, applying accelerated ageing is very hazardous if the mechanisms involved under real conditions are not well known. Moreover, it is well known that the nature of the water can play a role, at least on diffusion kinetics [21]. The aim of this study is to improve the knowledge of the involved mechanisms and to propose reliable accelerated protocols. Accelerated ageing was performed on a silica filled polychloroprene in natural renewed sea water at 20, 40, 60 and $80^{\circ} \mathrm{C}$ for durations up to two years. The evolutions of chemical and mechanical properties were investigated to assess the ageing mechanisms and their consequences. Finally, specimens cut from a sample aged under service conditions for 23 years (and for the same compound formulation) were characterized in the same manner. In the discussion section, the data obtained from mechanical and physical analysis are combined for samples aged under accelerated and natural conditions in order to provide precious data on ageing mechanisms and to challenge the ageing protocols proposed.

\section{Methods}

\subsection{Material}

The material studied is a silica-filled polychloroprene used for the external layer of offshore floating flow lines. Its main components and mechanical characteristics are given in table 1 and 2 . It is worth noting that there is no silane coupling agent in the formulation. Square plates of $2 \mathrm{~mm}$ thickness and $250 \mathrm{~mm}$ width were manufactured by an industrial partner from the same material batch, in order to ensure the reliability of mixing and moulding conditions. 


\begin{tabular}{|c|c|}
\hline Coumpond & phr \\
\hline Rubber & 100 \\
MgO & 4 \\
ZnO & 6 \\
Stearic Acid & 2 \\
Accelerators & 1 \\
Plasticizer & less than 10 \\
Carbon Black & 6 \\
Silica & 46 \\
Antioxidant & 2 \\
\hline
\end{tabular}

Table 1: Polychloroprene formulation

\begin{tabular}{|c|c|}
\hline Properties & CR \\
\hline Density & 1.4 \\
Hardness (Shore A) & 68 \\
Stress at break [MPa] & 17.4 \\
Elongation at break [\%] & 670 \\
\hline
\end{tabular}

Table 2: Mechanical properties of the chloroprene rubber

\subsection{Accelerated Ageing}

Specimens were immersed in several tanks filled with natural sea water coming directly from the Brest estuary and maintained at different temperatures: 20, 40, 60 and $80^{\circ} \mathrm{C}$. Water was continually renewed using a peristaltic pump leading to the replacement of the vessel volume (60L) every 24 hours without any modification of the temperature. $2 \mathrm{~mm}$ thick plates were removed periodically from the tanks and subsequently dried at $40^{\circ} \mathrm{C}$ in an inert atmosphere until a constant weight was reached. This drying protocol was optimised and validated by a specific study detailed elsewhere [22].

\subsection{Natural ageing}

The naturally aged sample comes from the coating layer of an offshore floating export line. This flow line was used in the Atlantic Ocean for 23 years at 10 meters depth and was still meeting the structural mechanical requirements after its withdrawal. Sea water temperature is about $15 \mathrm{C}$ to $25 \mathrm{C}$ in this area and $\mathrm{pH}$ is around 8.2 . The $2.3 \mathrm{~mm}$ thick topcoat, dedicated 
to protect the structure from environmental degradation, did not reveal any damage after its service life. The topcoat was made to the same recipe as the samples used for the accelerated ageing tests.

\subsection{Tensile Test}

After the ageing and drying steps the samples were cut from the sheets with a punch die in order to obtain normalised dumbbell shapes (type 2 from the standard ISO 37:2005). The tensile tests were performed on a Lloyd LR5K + testing machine, with a $1 \mathrm{kN}$ load cell. The tests were displacement controlled with a grip speed of $10 \mathrm{~mm} / \mathrm{min}$. A laser extensometer LASERSCAN200 was used to measure the local elongation. For each ageing condition, at least three samples were tested and the results averaged.

\subsection{Elastic modulus profiling}

The elastic modulus profiles were obtained using a CSM-instruments micro-hardness tester with a Vickers tip. The samples cut out from $2 \mathrm{~mm}$ thick sheets were embedded at room temperature in epoxy resin and subsequently ground with grinding media of decreasing granulometry (down to grit size 800) in wet conditions. The disc rotation speed was low in order to limit the possible rise of temperature. After grinding, a delay of 2 hours was systematically applied in order to let the samples cool down to ambient temperature. All tests were load controlled. A classical testing protocol was used, with a low preload in order to detect the surface contact (set to 10 $\mathrm{mN}$ ), then a load controlled loading step (up to $100 \mathrm{mN}$ at a loading rate of $200 \mathrm{mN} / \mathrm{min}$ ), followed by a creep test of $30 \mathrm{~s}$ and then a load controlled unloading step (down to $0 \mathrm{mN}$ at a unloading rate of $200 \mathrm{mN} / \mathrm{min}$ ). Each point is an average of 5 measurements. An instrumented hardness was evaluated from the load-displacement curve using the following equation :

$$
H_{I T}=\frac{F_{\max }}{A_{p}}
$$

where $A_{p}$ is the projected area of the contact surface evaluated from the Oliver and Pharr methodology [23]. In the following, we will focus only on the normalized hardness, i.e. the value of the hardness divided by its initial value obtained on the unaged material. This choice is motivated by the fact that this micro-hardness quantity is strongly dependant on the affected 
volume. Therefore, its value can not be easily correlated to more classical hardness values such as macroscopic Shore and DIDC hardness, but the variation of hardness can be easily interpreted.

\subsection{FTIR}

FTIR characterization was performed with a Thermo Nicolet spectrometer using a $4 \mathrm{~cm}^{-1}$ resolution and 32 scans. When ATR mode was used, spectra were normalized using the $\mathrm{C}-\mathrm{Cl}$ bond situated at $825 \mathrm{~cm}^{-1}$ [14]. The stability of this bond throughout ageing was confirmed using NMR measurements. 50 micros films were used to generate the chemical profiles through the samples thickness, using transmission measurements. The films were cut from samples, cooled by liquid nitrogen, with a Leica microtome.

\subsection{Solid state NMR}

Samples from $2 \mathrm{~mm}$ thick samples were ground into powder in liquid nitrogen. All NMR experiments were conducted at room temperature on a Bruker Avance $500 \mathrm{MHz}$ spectrometer. ${ }^{1} \mathrm{H}$ and ${ }^{13} \mathrm{C}$ MAS spectra were acquired at a MAS frequency of $10 \mathrm{kHz}$ using a $4 \mathrm{~mm}$ MAS probehead. In both cases, recycle delay was $2 \mathrm{~s}$. For ${ }^{13} \mathrm{C}$ spectra, $1 \mathrm{H}$ decoupling (spinal64) with a RF field of $60 \mathrm{kHz}$ was used during acquisition. Both ${ }^{29} \mathrm{Si} \mathrm{MAS}$ and ${ }^{1} \mathrm{H}_{-}{ }^{29} \mathrm{Si}$ ramped CP-MAS spectra were acquired on a $7 \mathrm{~mm}$ MAS probe using a MAS frequency of $5 \mathrm{kHz}$ and spinal64 $1 \mathrm{H}$ decoupling of $60 \mathrm{kHz}$ strength. Recycle delays were set to $5 \mathrm{~s}$ and $3 \mathrm{~s}$ for MAS and CPMAS experiments, respectively. ${ }^{1} \mathrm{H}_{-}{ }^{29} \mathrm{Si} \mathrm{CP}-\mathrm{MAS}$ spectra were obtained with a $1 \mathrm{~ms}$ contact time. ${ }^{13} \mathrm{C},{ }^{1} \mathrm{H}$ and ${ }^{29} \mathrm{Si}$ chemical shifts are referenced to TMS at $0 \mathrm{ppm}$.

\section{Results}

\subsection{Accelerated Ageing}

\subsubsection{Tensile properties}

Figure 1 shows the evolution of the tensile behavior of the material after accelerated ageing at $60^{\circ} \mathrm{C}$ for different durations. Ageing leads to a significant increase of the initial modulus (up to $200 \mathrm{MPa}$ after 6 months) and a considerable decrease of ultimate strain and stress values. Figure 2 shows that these evolutions are observed for all ageing temperatures with a strong effect of temperature on the degradation kinetics. It can also be concluded that for each temperature, the longer the duration of ageing, the more significant the changes in tensile properties. 


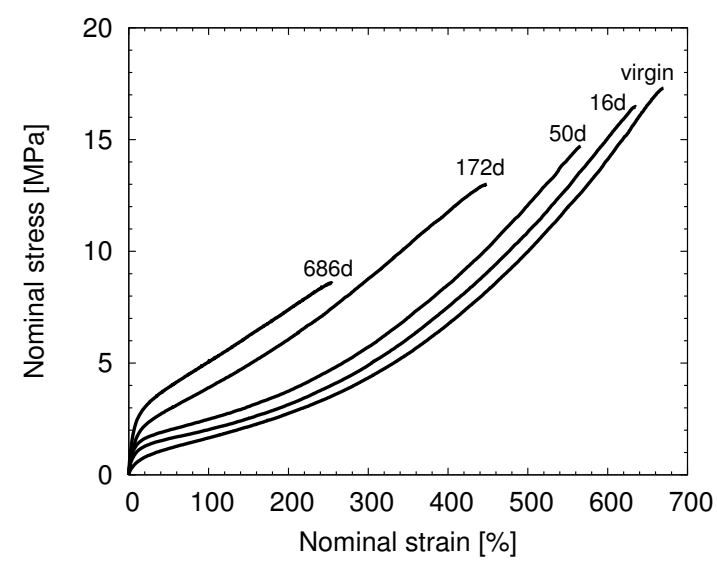

Figure 1: Tensile behavior evolution after ageing at $60^{\circ} \mathrm{C}$ in sea water for different periods (d for days)

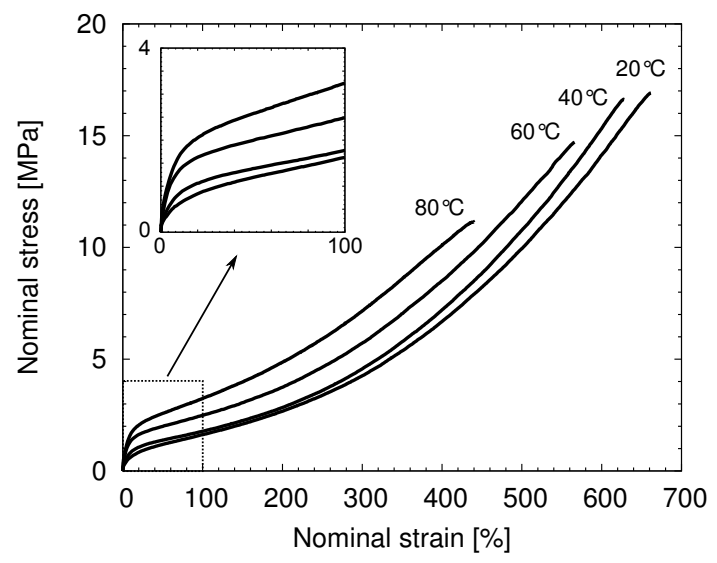

Figure 2: Stress versus strain curves after 50 days of ageing at different temperatures

\subsubsection{Elastic modulus profiles}

In order to simplify the evaluation of the modulus evolution, the variable considered in this section is the ratio between the elastic modulus and the modulus measured on an un-aged material. Figures 3 and 4 plot the values measured across the sample thickness after accelerated ageing for several ageing temperatures and durations. Whatever the ageing conditions, the aged samples always exhibit a higher external stiffness. A clear gradient between the skin and the core is also observable, which is stronger as the ageing is more severe (i.e. higher temperatures and/or longer durations). 


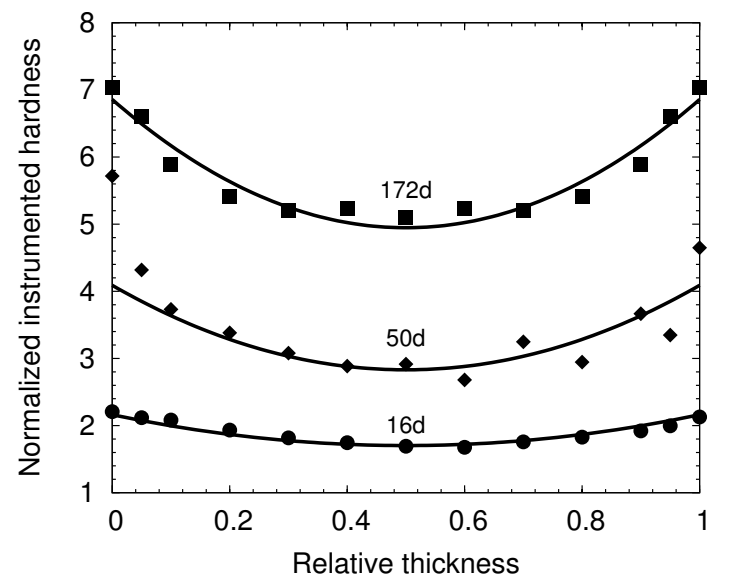

Figure 3: Evolution of the normalized hardness through the sample thickness for different ageing durations at $80^{\circ} \mathrm{C}$

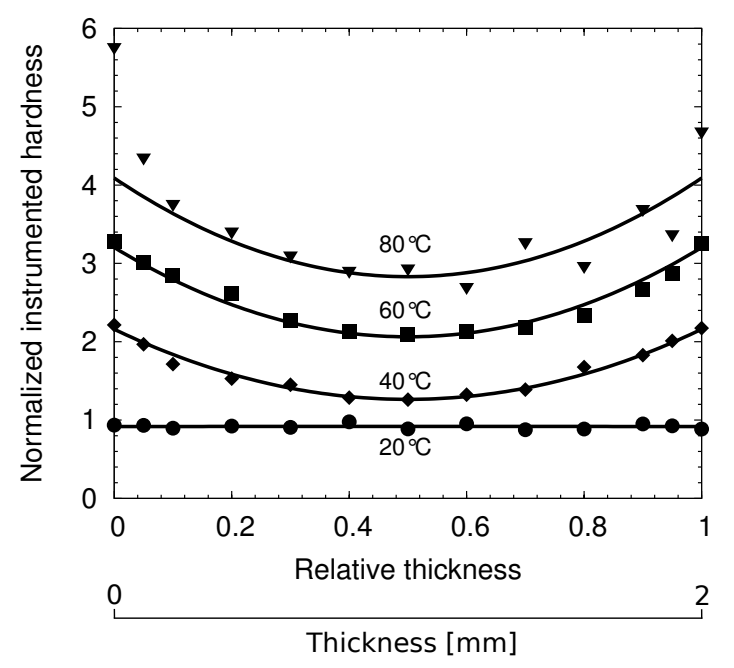

Figure 4: Evolution of the normalized hardness profile through the sample thickness for all ageing temperature after 50 days of ageing

\subsubsection{FTIR}

Figure 5 shows the FTIR spectra for the unaged polychloroprene; the band attributions are given in table 3 . As illustrated on figure 6 , the intensity of the $1100 \mathrm{~cm}^{-1}$ peak (attributed to the Si-O bond of the silica filler [24]) decreases significantly during ageing. At the same time, a new peak located at $1014 \mathrm{~cm}^{-1}$ appears and rises according to the ageing duration. The exact 
attribution of this new band is not clear but could be related to the formation of $\mathrm{SiOH}, \mathrm{C}-\mathrm{OH}$ or $\mathrm{C}-\mathrm{C}$ bonds [2], [24].

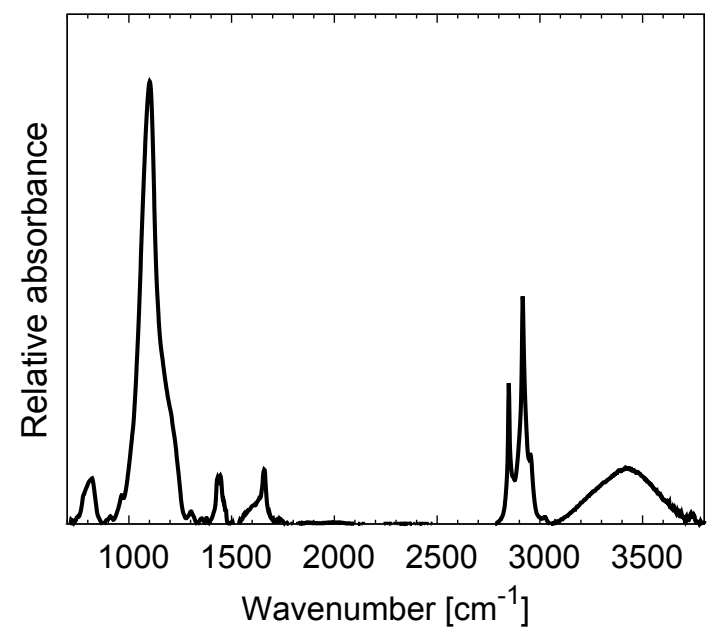

Figure 5: FTIR spectra of the unaged polychloroprene

\begin{tabular}{|c|c|}
\hline WaveNumber $\left(\mathrm{cm}^{-1}\right)$ & Band Assignment \\
\hline 825 & $\mathrm{C}-\mathrm{Cl}$ \\
\hline 1100 & $\mathrm{Si}-\mathrm{O}$ \\
\hline 1450 & $\mathrm{CH}$ \\
\hline 1660 & $\mathrm{C}=\mathrm{C}$ \\
\hline 2848 & $\mathrm{CH}_{2}$ symmetric \\
\hline 2917 & $\mathrm{CH}_{2}$ asymmetric \\
\hline 3450 & $\mathrm{OH}$ \\
\hline
\end{tabular}

Table 3: Band Assignments

\subsubsection{NMR}

Solid state NMR characterization has been performed to understand the mechanisms involved when $\mathrm{CR}$ is immersed in sea water. ${ }^{1} \mathrm{H}$ and ${ }^{13} \mathrm{C}$ measurements have been made in order to evaluate the evolution of the polychloroprene backbone. ${ }^{29} \mathrm{Si}$ measurements have been used to evaluate the silica filler modification during ageing. 


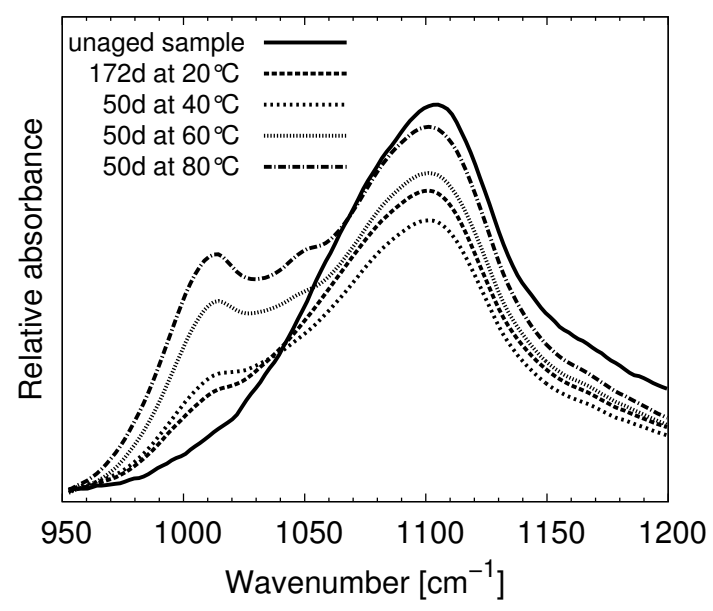

Figure 6: FTIR evolution during ageing in sea water at different temperatures

${ }^{1} \mathbf{H}$ and ${ }^{13} \mathbf{C}$ NMR Figure 7 compares the ${ }^{1} \mathrm{H}$ MAS spectra of the unaged sample and aged sample, band assignments are given in table 4 .

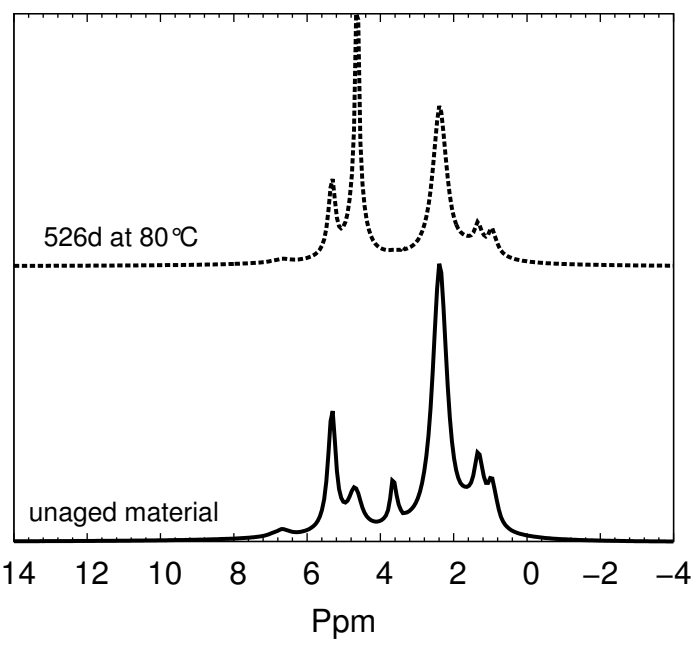

Figure 7: ${ }^{1} \mathrm{H}$ MAS spectra for unaged and aged CR

Figure 8 compares the ${ }^{13} \mathrm{C}$ MAS spectra of the unaged sample with those of samples aged under different conditions, band assignments are given in table 5 .

Except for the lines attributed to 1,2 isomerized $\mathrm{CR}\left(3.7 \mathrm{ppm}\right.$ in ${ }^{1} \mathrm{H}$ and 58.0 in ${ }^{13} \mathrm{C}$ ), there is no evolution of ${ }^{1} \mathrm{H}$ and ${ }^{13} \mathrm{C}$ spectra after 526 days at $80^{\circ} \mathrm{C}$ 


\begin{tabular}{|c|c|}
\hline Signal (ppm) & Band Assignment \\
\hline $1.0 \& 1.3$ & $\begin{array}{c}\text { Free hydroxyls } \\
\text { of silanol }\end{array}$ \\
\hline $2.4 \& 5.3$ & $\begin{array}{c}\text { H1/H4 and H3 } \\
\text { of the } 1,4 \text { trans } \\
\text { polymerization }\end{array}$ \\
\hline 3.7 & Isomerized $1,2 \mathrm{CR}$ \\
\hline 4.7 & Water \\
\hline 6.7 & H-bonded silanol \\
\hline
\end{tabular}

Table 4: ${ }^{1} \mathrm{H}$ MAS signal assignment for CR

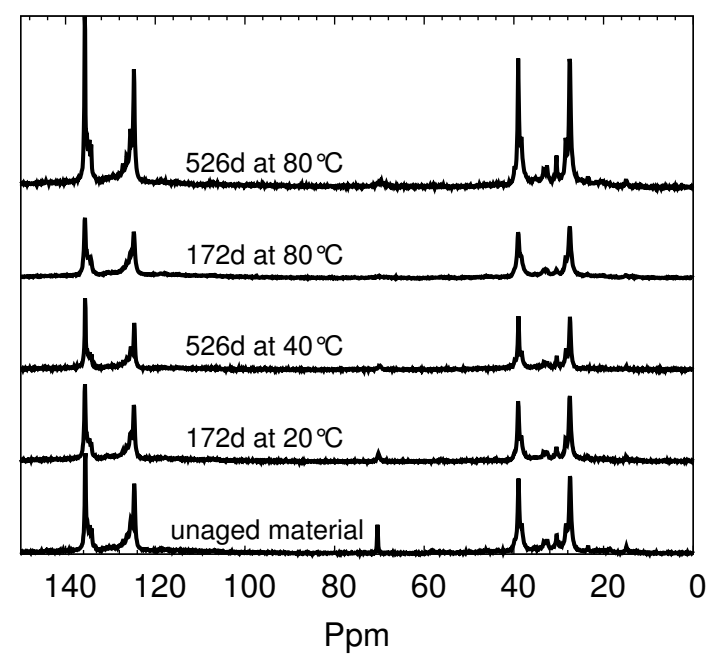

Figure 8: ${ }^{13} \mathrm{C}$ MAS spectra for unaged and aged CR

in sea water. This is an obvious indication of the polychloroprene backbone stability during ageing. These results clearly validate the utilization of the $\mathrm{C}-\mathrm{Cl}$ bond as an internal standard for FTIR spectra. The signal situated at $70 \mathrm{ppm}$ in ${ }^{13} \mathrm{C}$ spectra can not be clearly assigned, in fact no signal in this chemical shift region has ever been reported in the literature for CR.

${ }^{29}$ Si NMR The ${ }^{29} \mathrm{Si}$ MAS spectrum of silica fillers (figure 9) exhibits 3 lines at -112, -102 and -92 ppm labeled A4, A3 and D3. ${ }^{29} \mathrm{Si}$ isotropic chemical shift is known to be sensitive to the dimensionality of silicate networks 


\begin{tabular}{|c|c|}
\hline Signal $(\mathrm{ppm})$ & Band Assignment \\
\hline 27.4 & 1,4 trans CR \\
& $\delta$ position \\
\hline 38.9 & 1,4 trans CR \\
& $\gamma$ position \\
\hline 58.0 & Isomerized $1,2 \mathrm{CR}$ \\
\hline 124.6 & 1,4 trans CR \\
& $\beta$ position \\
\hline 135.5 & 1,4 trans CR \\
& $\alpha$ position \\
\hline
\end{tabular}

Table 5: ${ }^{13} \mathrm{C}$ MAS signal assignment for $\mathrm{CR}$

[25], [26]. Thus, $\mathrm{Qn}_{\mathrm{SiO}_{4}}$ species $\left(\mathrm{Q}_{n}=\mathrm{Si}(\mathrm{OSi})_{n}(\mathrm{OH})_{4-n}\right)$ typically fall in the $-116 /-100,-105 /-90$ and $-95 /-75$ ppm ranges for $\mathrm{n}=4,3$ and 2 , respectively. So the A4 line can be unambiguously assigned to Q4 species. The strong enhancement observed on the A3 line intensity by CP technique clearly shows that it corresponds to the Q3 species. The D3 case is somewhat more delicate since its isotropic chemical shift falls inside the Q2 and Q3 shift ranges simultaneously. Nevertheless, the D3/A3 intensity ratio is exactly the same on the MAS and CPMAS spectra. So, we can also attribute D3 to Q3 species. After addition of silica fillers in chloroprene rubber (unaged sample in figure 10), we can observe the appearance of a line at $-86 \mathrm{ppm}$ (labeled A2) assigned to Q2. At the same time, an evolution of intensity ratio is observed : A3/A4 increases whereas D3/A4 decreases. Moreover, the D3/B3 intensity ratio is the same in MAS and CPMAS. These observations could be explained by a modification of the $\mathrm{OH}$ environment (for Q3 and Q2 species) due to interaction with the CR backbone, but also by a small depolymerisation of the silica network during processing.

During ageing, a gradual increase of the D3/A4 and A2/A4 ratios is observed as shown in figure 10. This evolution is a clear indication of the silanol formation from the silica filler during ageing and it is in agreement with the decrease of the $1100 \mathrm{~cm}^{-1}$ band and the appearance of the 1014 $\mathrm{cm}^{-1}$ band in FTIR spectra.

NMR results show that the CR backbone is not greatly modified during ageing in sea water whereas the silica undergoes degradation leading to the formation of silanol. This behavior is observed for all the ageing temperatures 


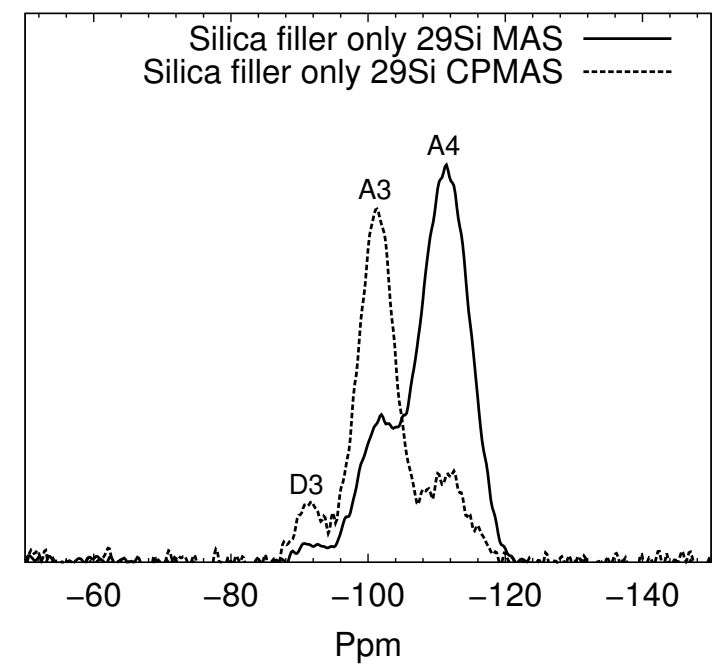

Figure 9: ${ }^{29} \mathrm{Si}$ MAS and CP-MAS spectra for silica filler

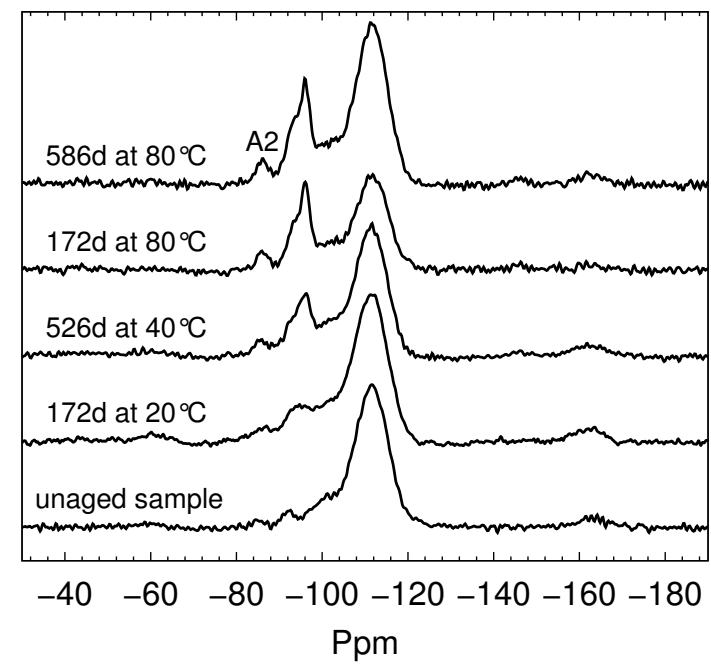

Figure 10: Evolution of ${ }^{29} \mathrm{Si}$ MAS spectra during ageing

used in this study, i.e. from $20^{\circ} \mathrm{C}$ to $80^{\circ} \mathrm{C}$.

\subsection{Natural Ageing}

\subsubsection{Tensile properties}

The tensile curves obtained for an un-aged sample and for a sample aged naturally for 23 years are plotted in figure 11. Natural ageing leads to a 
significant rise of the initial stiffness and a strong decrease of the elongation at break. These consequences are indeed very similar to the ones observed for accelerated ageing.

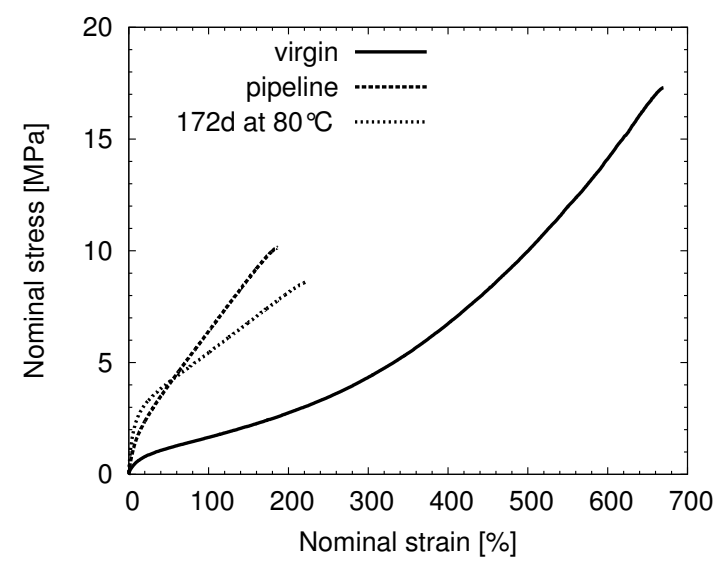

Figure 11: Tensile behavior of the naturally aged sample

\subsubsection{Elastic modulus profiles}

Here again, the ratio between the elastic modulus and the modulus measured on an un-aged material is considered. Figure 12 plots the values measured through the sample thickness after natural ageing. Only the external face (left) was exposed to water, which explains the asymmetrical profile obtained. It is worth noting that the value on the internal face is comparable to the value measured on unaged samples. The naturally aged sample exhibits a clear gradient between the skin and the core with a very significant increase of the elastic modulus on the side exposed to water (left side in figure 12). No cracks were observed on the surface sample after 23 years of ageing, the hardness profile through the thickness is probably due to reaction/diffusion phenomena.

\subsubsection{FTIR}

FTIR measurements were performed on the sample aged naturally for 23 years at different distances from the surface. Figure 13 illustrates the FTIR spectra observed at the surface, with a clear appearance of a new band at $1720 \mathrm{~cm}^{-1}$ due to the carbonyl formation during CR oxidation [14], [27]. This oxidation is limited to the first 200 microns from the external layer and thus can not explain the modulus profile observed in figure 12 Different hypotheses 


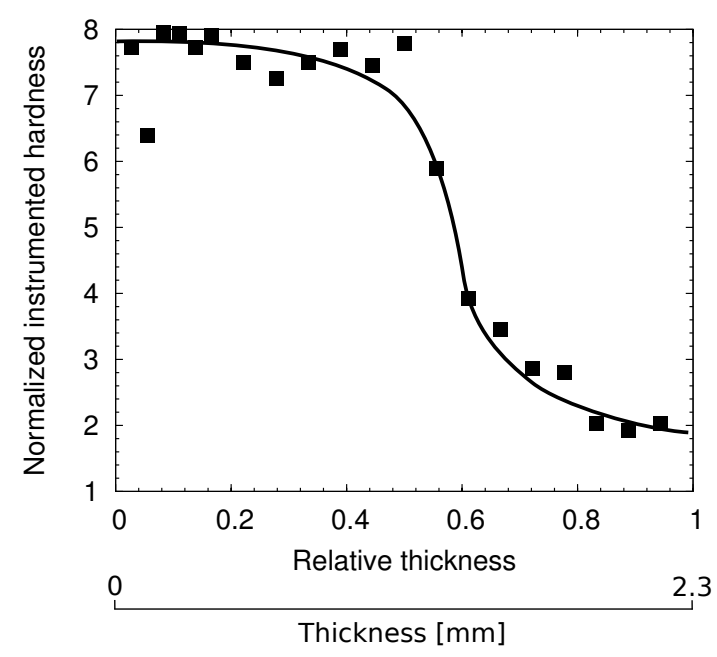

Figure 12: Evolution of the hardness in the thickness of the naturally aged sample

can be made to explain this depth limitation. First, CR oxidation by UV, it is well known that the UV oxidation is limited to a few microns from the surface due to a strong absorption of the UV energy by the polymer [28], [29]. The observed ketone formation could also be due to a reaction of the polymer with ozone; this kind of reaction is also located close to the external surface [30]. And finally, a diffusion of antioxidant from the material into water can be assumed [12],[31], this decrease of the stabilization close to the surface can also lead to a local oxidation of the material.

Focus on the $900-1200 \mathrm{~cm}^{-1}$ region $900-1200 \mathrm{~cm}^{-1}$ (Figure 14) clearly shows that the $1100 \mathrm{~cm}^{-1}$ peak has almost disappeared after ageing, whereas the $1014 \mathrm{~cm}^{-1}$ peak has increased.

\section{Discussion}

\subsection{Consequences and mechanisms of degradation}

The mechanical tests after both natural and accelerated ageing clearly show common features: a strong increase in the material stiffness (for tensile tests and micro-hardness measurements) and a large drop in failure properties. It is worth noting that for severe ageing, a strong gradient is visible on the aged samples, so very careful extrapolation of the values obtained from tensile samples is required, as they are no longer homogeneous. Three main explanations are usually found in the literature for such mechanical 


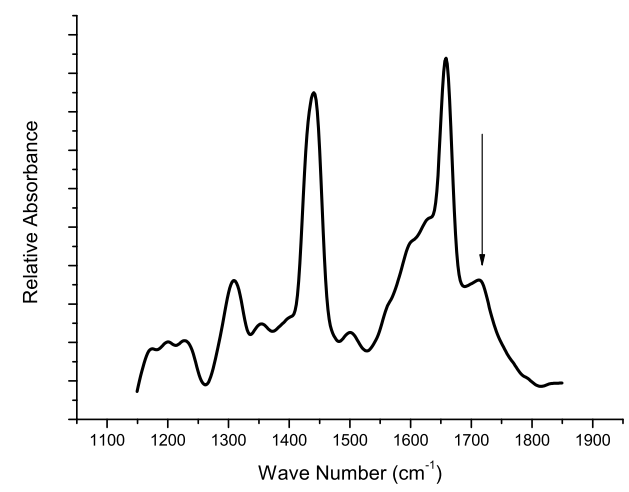

Figure 13: FTIR spectra of the surface of naturally aged sample

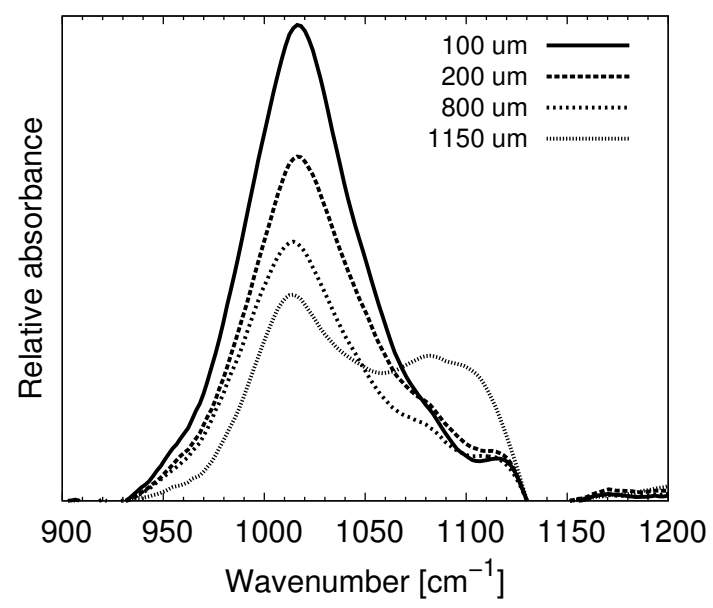

Figure 14: Evolution of FTIR results as a function of depth in the naturally aged sample

changes : loss of plasticizers, oxidation and crosslink creation. The amount of plasticizers is too low here to be the only explanation [32], [33]. Oxidation is sometimes proposed as a relevant mechanism to explain marine ageing [13] but the results of FTIR and NMR measurements clearly show that the classic peaks were not found here, for either natural or accelerated ageing. An explanation based on an increase of the crosslink density therefore seems to be relevant. For polychloroprene, a first idea is related to the chlorine consumption, which would create very reactive double $\mathrm{C}=\mathrm{C}$ links. The ${ }^{13} \mathrm{C}$ NMR measurements clearly shows that the peak associated to the $\mathrm{C}-\mathrm{Cl}$ link 
is not evolving, and can be used as a reference to normalize the FTIR spectra. Moreover, this technique also shows that the carbon signature of the polychloroprene did not evolve much during ageing. What is highlighted by the chemical measurements is the formation of silanol with ageing, for natural and accelerated samples. This formation is due to silica hydrolysis often described in literature [34]. Whereas the silica hydrolysis is a normally an equilibrated reaction [34],[35] leading to silanol recombination, in this case no Si-O formation is observed. This could be explained by different factors, such as a high concentration of water in the rubber (more than $10 \mathrm{~mol} / \mathrm{L}$ ) [36], the $\mathrm{pH}$ of the sea water (8.2) [35], but also the fact that silanol is more hydrophilic than silica [37] leading to a local increase in the water concentration and thus modify the equilibrium of the reaction. It also appears that the silanol concentration increases with ageing severity (higher water temperature and/or duration). The intensity of the silanol FTIR peak is therefore very likely to be useful as a local indicator of marine ageing for this silica filled polychloroprene. In order to assess this assumption, the profiles obtained from FTIR measurements and micro-hardness measurements are compared for accelerated ageing (figure 15) and for natural ageing (figure $16)$.
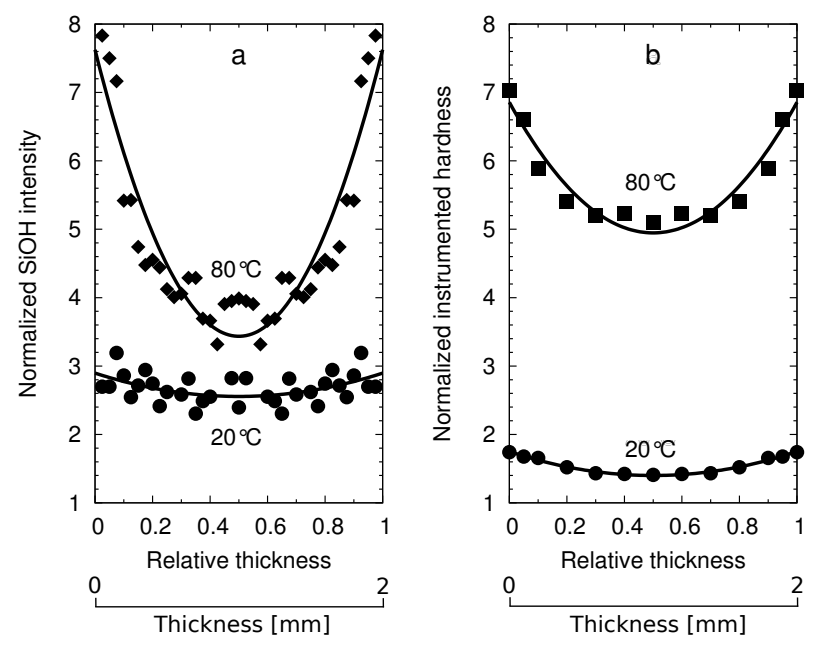

Figure 15: Correlation between a) Si-OH profiles and b) the stiffness profiles for samples aged in the laboratory at $20^{\circ} \mathrm{C}$ and $80^{\circ} \mathrm{C}$ for 172 days

The conclusion is that there is a strong correlation between silanol concentration and micro hardness for both kinds of aged samples. It is therefore 


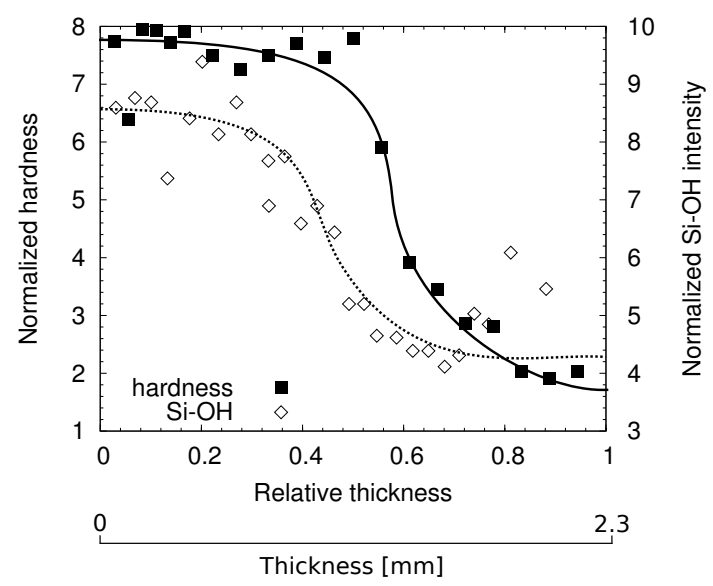

Figure 16: Correlation between the stiffness and Si-OH profiles for the naturally aged sample

clear that there is a link between the silanol formation and the increase in the local elastic modulus. Nevertheless, the associated crosslinking process is still not clear. Some authors [38] [39] have suggested the existence of hydrogen bonds between the chlorine atom of the polychloroprene and the hydrogen atom of the silanol as represented in figure 17.

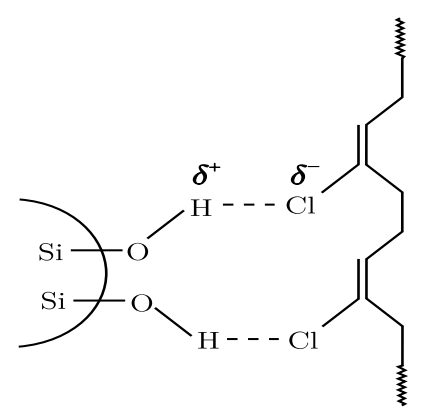

Figure 17: Interaction between CR and silica (H-bonds)

Other mechanisms may contribute to the material stiffening, such as a better filler distribution in the material or the formation of a silica network by silanol condensation. Nevertheless, those mechanisms would be unlikely to lead to such large effects.

The degradation profile through the sample thickness after 172 days at $80^{\circ} \mathrm{C}$, shown on figure 15 is an indication of a diffusion/reaction phenomenon. This 
kind of behaviour is well known for elastomer oxidation [40], [41] and can be explained by the competition between degradation and diffusion. For the ageing considered here, at low temperature water diffusion is faster than silica hydrolysis which leads to a homogeneous degradation in the sample thickness, as shown in figure 15. At higher temperatures, the kinetics of silica hydrolysis are faster than the water diffusion, leading to the degradation profiles observed.

\subsection{Comparison between natural and accelerated ageing}

The comparison between the mechanical and chemical measurements (figure 16) clearly illustrates that the accelerated ageing is caused by the same ageing mechanisms as the natural ageing, i.e. silica hydrolysis, and leads to the same mechanical consequences, i.e. a stiffening of the material, from a local or global point of view, and a drop in the failure properties. The ageing protocol which consists of using hot, renewed natural, sea water to accelerated polymer degradation is therefore appropriate to study marine ageing in the case of this silica-filled polychloroprene. It is important to underline that, in a similar manner to the studies dealing with oxidation, the identification of the diffusion, consumption and degradation kinetics are crucial to perform the validation on aged structures, as the gradients observed are strongly dependent on the temperature and on the geometry. In fact, differences between modulus profiles observed after accelerated and natural ageing (figures 12 and 15) are mainly due to differences in sample thickness and boundary conditions. The mechanical loading of the flow line could change water diffusion in the polymer or kinetics of chemical reactions and thus lead to a modification of the modulus profile in the sample.

\section{Conclusion}

The ageing in seawater of a silica-filled polychloroprene rubber (CR) has been investigated. The CR degradation has been analysed in order to highlight the chemical mechanisms and the mechanical consequences for both accelerated and long term natural ageing. Whereas the silica hydrolysis has been published many times, the effect of this hydrolysis on chloroprene rubber properties has never been detailed. The mechanical consequences are similar for natural and accelerated ageing, i.e. a strong increase of the material stiffness (observed for tensile tests and micro-hardness measurements) and a large drop in failure properties. The combined results of FTIR and 
solid state NMR show that during marine ageing, the silica used as filler in the rubber undergoes hydrolysis, resulting in silanol formation. The amount of silanol seems to be a relevant chemical indicator of the ageing mechanisms and the comparison of the modulus and silanol profiles indicates a strong correlation, very likely explained by the creation of hydrogen bonds between hydroxyl and chlorine atoms. For severe ageing, strong gradients in mechanical and chemical properties are visible on the aged samples, which must be taken into account in a lifetime prediction. Two main perspectives will be investigated in forthcoming studies: full investigation of the kinetics of water diffusion and silica hydrolysis by using a mechanistic approach, in order to predict the ageing gradients; further measurements in order to understand the link between the silanol concentration and the changes in mechanical properties. These studies will clearly benefit from the validation of the use of hot natural renewed seawater to speed up the polymer degradation in a marine environment.

\section{Ackowledgements}

The authors would like to thank the Brittany region for financial support, all the actors involved in the FEMEM project for helpful discussions, Elodie Breton for performing numerous measurements and Peter Davies for the careful reading of the paper.

\section{References}

\section{References}

[1] Guermazi N, Elleuch K, Ayedi H. The effect of time and aging temperature on structural and mechanical properties of pipeline coating. Materials \& Design 2009;30(6):2006-10. doi:doi: DOI: 10.1016/j.matdes.2008.09.003.

[2] Davies P, Evrard G. Accelerated ageing of polyurethanes for marine applications. Polymer Degradation and Stability 2007;92(8):1455 -64. doi:DOI: 10.1016/j.polymdegradstab.2007.05.016.

[3] Legghe E, Aragon E, Blec L, Margaillan A, Melot D. Correlation between water diffusion and adhesion loss: Study of an epoxy primer on steel. Progress in Organic Coatings 2009;66(3):276 -80. doi:DOI: 10.1016/j.porgcoat.2009.08.001. 
[4] Rutkowska M, Krasowska K, Heimowska A, Steinka I, Janik H. Degradation of polyurethanes in sea water. Polymer Degradation and Stability 2002;76(2):233 -9. doi:10.1016/S0141-3910(02)00019-8.

[5] Unsworth J, Ng Y. Effects of immersion in sea water on the properties of polymers used in special marine cable. Internationnal conference on Polymers in a marine environments 1984;97(Conf 2, paper 10):73-6.

[6] Ab-Malek K, Stevenson A. The effect of 42 year immersion in seawater on natural rubber. Journal of Materials Science 1986;21:147-54. 10.1007/BF01144713.

[7] Pegram JE, Andrady AL. Outdoor weathering of selected polymeric materials under marine exposure conditions. Polymer Degradation and Stability 1989;26(4):333 -45. doi:DOI: 10.1016/0141-3910(89)90112-2.

[8] Oldfield D, Symes T. Long term natural ageing of silicone elastomers. Polymer Testing 1996;15(2):115 -28. doi:DOI: 10.1016/01429418(95)00018-6.

[9] Bowditch M, Stannard K. Effect of water absorption on the properties of a filled elastomer. Internationnal conference on Polymers in a marine environments 1984;97(Conf 2, paper 17):117-9.

[10] Merdas I, Thominette F, Verdu J. Hydrolytic ageing of polyamide 11-effect of carbon dioxide on polyamide 11 hydrolysis. Polymer Degradation and Stability 2003;79(3):419 -25. doi:DOI: 10.1016/S01413910(02)00358-0.

[11] Belan F, Bellenger V, Mortaigne B, Verdu J. Relationship between the structure and hydrolysis rate of unsaturated polyester prepolymers. Polymer Degradation and Stability 1997;56(3):301 -9. doi:DOI: 10.1016/S0141-3910(96)00203-0.

[12] Geertz G, Brll R, Wieser J, Maria R, Wenzel M, Engelsing K, et al. Stabiliser diffusion in long-term pressure tested polypropylene pipes analysed by ir microscopy. Polymer Degradation and Stability 2009;94(7):1092 -102. doi:10.1016/j.polymdegradstab.2009.03.020.

[13] Mott P, Roland C. Aging of natural rubber in air and seawater. Rubber Chemistry and Technology 2001;74(1):79-88. 
[14] Celina M, Wise J, Ottesen DK, Gillen KT, Clough RL. Correlation of chemical and mechanical property changes during oxidative degradation of neoprene. Polymer Degradation and Stability 2000;68(2):171 -84. doi: DOI: 10.1016/S0141-3910(99)00183-4.

[15] Ha-Anh T, Vu-Khanh T. Prediction of mechanical properties of polychloroprene during thermo-oxidative aging. Polymer Testing 2005;24(6):775 -80. doi:DOI: 10.1016/j.polymertesting.2005.03.016.

[16] Burns J, Dubbelday PS, Ting RY. Dynamic bulk modulus of various elastomers. Journal of Polymer Science Part B: Polymer Physics 1990;28(7):1187-205. doi:10.1002/polb.1990.090280715.

[17] Samay G, Palotas L, Nagy T, Seregely Z. Lifetime-affecting factors of rubber products. Journal of Applied Polymer Science 1992;50:359-67.

[18] Leveque X. Behaviour of various elastomers in a marine environment. Internationnal conference on Polymers in a marine environments 1984;97(Conf 2, paper 16):111-5.

[19] Verdu J. Action de l'eau sur les plastiques. Techniques de l'ingnieur 2000;(AM3165).

[20] Hinkley JA, Holmes BS. Effect of water on the strength of filled polychloroprene vulcanizates. Journal of Applied Polymer Science 1986;32(5):4873-81. doi:10.1002/app.1986.070320510.

[21] Choqueuse D, Chomard A, Bucherie C. Insulation materials for ultra deep sea flow assurance : Evaluation of the material properties. In: Proceedings of Offshore Technology Conference. 2002,doi:10.4043/14115MS.

[22] Le Saux V, Marco Y, Calloch S, Le Gac PY. Marine ageing of polychloroprene rubber: validation of accelerated protocols and static failure criteria by comparison to a 23 years old offshore export line. ECCMR conference 2011;

[23] Oliver W, Pharr G. An improved technique for determining hardness and elastic modulus using load and displacement sensing indentation experiments. Journal of Materials Research 1992;:1564-83doi: 10.1557/JMR.1992.1564. 
[24] Socrates G. Infrared and raman characteristic group frequencies : tables and charts. West Sussex: John Wiley \& Sons Inc.; 3. ed.paperback ed., repr. ed.; 2007. ISBN 9780470093078.

[25] Lippmaa E, Maegi M, Samoson A, Engelhardt G, Grimmer AR. Structural studies of silicates by solid-state high-resolution silicon-29 $\mathrm{nmr}$. Journal of the American Chemical Society 1980;102(15):4889-93. doi: 10.1021/ja00535a008.

[26] MacKenzie K. Multinuclear solid-state NMR of inorganic materials. Oxford New York: Pergamon; 1st ed. ed.; 2002. ISBN 9780080437873.

[27] Delor F, Lacoste J, Lemaire J, Barrois-Oudin N, Cardinet C. Photo- and thermal ageing of polychloroprene: Effect of carbon black and crosslinking. Polymer Degradation and Stability 1996;53(3):361 -9. doi:DOI: 10.1016/0141-3910(96)00097-3.

[28] Mallgol J, Gonon L, Lemaire J, Gardette JL. Long-term behaviour of oil-based varnishes and paints 4 . influence of film thickness on the photooxidation. Polymer Degradation and Stability 2001;72(2):191 -7. doi:10.1016/S0141-3910(00)00170-1.

[29] Girois S, Delprat P, Audouin L, Verdu J. Oxidation thickness profiles during photooxidation of non-photostabilized polypropylene. Polymer Degradation and Stability 1997;56(2):169 -77. doi:10.1016/S01413910(96)00175-9.

[30] Rodrigues FHA, Santos EF, Feitosa JPA, Ricardo NMPS, de Paula RCM. Ozonation of unstretched natural rubber: Part i. effect of film thickness. Rubber Chemistry and Technology 2001;74(1):57-68.

[31] Allen NS, Palmer SJ, Marshall G, Luc-Gardette J. Environmental oxidation processes in yellow gas pipe: implications for electrowelding. Polymer Degradation and Stability 1997;56(3):265 -74. doi:10.1016/S01413910(96)00148-6.

[32] Wypych G. Handbook of plasticizers. 2004.

[33] Akhter A FBMC. The effects of plasticizer and filler on the curing characteristic ans mechanical properties of (sbr) styrene butadiene rubber 
and (cr) chloroprene rubber blends. Journal of the Chemical Society of Pakistan 2008;30(4):507-11.

[34] Bunker B. Molecular mechanisms for corrosion of silica and silicate glasses. Journal of Non-Crystalline Solids 1994;179:300 -8. doi:DOI: 10.1016/0022-3093(94)90708-0. Proceedings of the First PAC RIM Meeting on Glass and Optical Materials.

[35] Brinker C. Hydrolysis and condensation of silicates: Effects on structure. Journal of Non-Crystalline Solids 1988;100(1-3):31-50. doi: 10.1016/0022-3093(88)90005-1.

[36] Le Saux V, Marco Y, Calloch S, Ait Hocine N, Le Gac PY. Accelerated ageing of polychloroprene for marine applications. Constitutive Models for Rubber VI 2009;

[37] Trouv A, Batonneau-Gener I, Valange S, Bonne M, Mignard S. Tuning the hydrophobicity of mesoporous silica materials for the adsorption of organic pollutant in aqueous solution. Journal of Hazardous Materials 2011;(0):. doi:10.1016/j.jhazmat.2011.11.043.

[38] Wang G, Li M, Chen X. Effects of fillers on mechanical properties of a water-swellable rubber. Journal of Applied Polymer Science 1999;72(4):577-84.

[39] Sae-oui P, Sirisinha C, Thepsuwan U, Hatthapanit K. Dependence of mechanical and aging properties of chloroprene rubber on silica and ethylene thiourea loadings. European Polymer Journal 2007;43(1):185 -93. doi:DOI: 10.1016/j.eurpolymj.2006.10.015.

[40] Colin X, Audouin L, Verdu J, Huy ML. Kinetic modelling of the thermal oxidation of polyisoprene elastomers. part 2: Effect of sulfur vulcanization on mass changes and thickness distribution of oxidation products during thermal oxidation. Polymer Degradation and Stability 2007;92(5):898 - 905. doi:DOI: 10.1016/j.polymdegradstab.2007.01.004.

[41] Wise J, Gillen KT, Clough RL. Time development of diffusion-limited oxidation profiles in a radiation environment. Radiation Physics and Chemistry 1997;49(5):565 -73. doi:DOI: 10.1016/S0969-806X(96)001855 . 